\title{
EXPERIMENTAL RESEARCH ON THE IMPACT OF THIN-WALL RATIO AND THE FILLET RADIUS OF FORMING ROLLER ON THE LIMITING SPINNING RATIO OF AMS 5504 SHEETS
}

\begin{abstract}
Results of experimental investigations of metal spinning process of AMS 5504 sheets. cylindrical drawpieces with use of discs-shaped sheet with various diameter and thickness were shown in this work. Tests were performed on two roller metal spinning machine of a vertical axis Leifeld SFC 800 V500.

The main objective of the study was to determine the dependence between limiting spinning coefficient, thin wall ratio coefficient and the fillet radius of forming rollers. To realize this aim required the development of an original methodology and experimental research plan. Determined relationship takes into account the occurrence of negative phenomena in the process of spinning such as wrinkling or cracking of material.

Due to the possibility of prediction of these negative phenomena, determined relationship is used in the design of a metal spinning processes. On the basis on determined relationship can be done a practical assessment of the possibility of shaping with certain geometrical parameters by spinning. Obtained results were shown graphically on the diagrams and their analysis was carried.

Keywords: spinning, experiment, AMS 5504 sheet, limiting spinning ratio, thin-wall ratio
\end{abstract}

\section{Introduction}

Sheet metal components of machines and devices can be produce by various methods including stamping. In the case of axisymetric drawpieces the alternative way is metal spinning and flow forming. These techniques are presented in detail in the scientific articles [1-2]. Their use is economically justified especially in the production of individual or small series. Therefore, the use of rotary methods is particularly useful in the production of axisymmetric drawpieces e.g. in the aerospace industry. Conventional spinning is the forming process, which allows perform of axi-symmetric products with simple or very complex shapes. As an input material for spinning can be used a flat disc or drawpiece. The conventional way of spinning of cylindrical drawpiece was shown in Fig. 1.

Disc (3) is centered and clamped with use of tailstock (2) to the face of the template (1) and rotates themselves with him. Roller (4) is forming the drawpiece in a single pass or multiple passes until a expected shape. In this type of spinning process sheet material is subjected to circumferential compressive and radial tensile stresses (Fig. 1). Such a stress scheme also occurs in drawing operations.

Depending on the type of spinning machine, the spinning process can be carried out by one or more rollers at the same time. In industry spinning machines most commonly is being used one or two rollers arranged opposite each other. Spinning

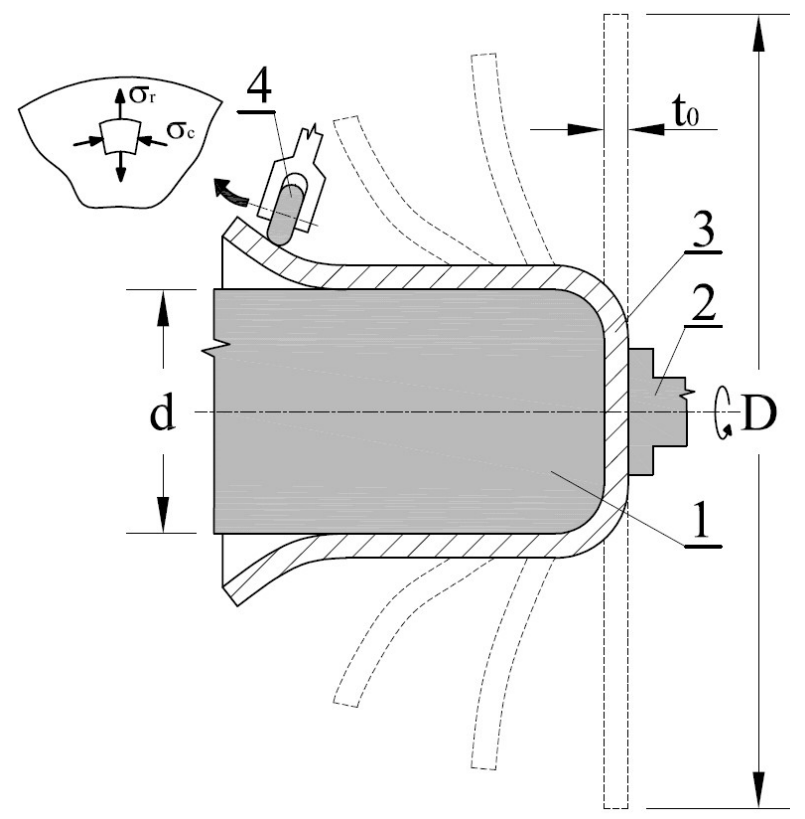

Fig. 1. Scheme of a conventional spinning process of cylindrical drawpiece: 1 - mandrel, 2 - tailstock, 3 - sheet, 4 - roller, D - initial diameter of the sheet, $d$-diameter of drawpiece, $t_{0}$ - initial thickness of sheet, $\sigma_{r}$ - radial stress, $\sigma_{c}-$ circumferential stress

with two rollers at the same time offers a number of advantages in comparison to the spinning with one roller. Among them we can mention: balancing the radial forces acting on the template to

\footnotetext{
* RZESZÓW UNIVERSITY OF TECHNOLOGY, 12 POWSTAŃCÓW WARSZAWY AV., 35-959 RZESZÓW, POLAND

** PRATT \& WHITNEY RZESZÓW S.A., 120 HETMAŃSKA , 35-078 RZESZÓW, POLAND

\# Corresponding author: stan_kut@prz.edu.pl
} 
prevent excessive radial load of bearing spindle and overcomes the adverse bending moment of template, increase efficiency and dimensional accuracy of products.

Sheet metal parts produced by spinning are used in the automotive, engineering and aerospace industry as well as works of art (vases, goblets, lamps, etc.), musical instruments and kitchen utensils (pots, trays, bowls, pans, funnels etc.). In this process, the components may be formed with a diameter of $3 \mathrm{~mm}$ to $10 \mathrm{~m}$ and a thickness of from 0.4 to $30 \mathrm{~mm}$ [3-4]. In the process of spinning can be shaped such materials as [5]: non-alloyed carbon steels, heat-resistant and stainless steels, non-ferrous heavy metals and light alloys.

Regardless of the spinning technique used during this process there may be negative phenomena limiting the ability to shape drawpieces in the process. They lead to defects on the products.

The most common defect in spinning are wrinkling (Fig. 2a), circumferential cracks (Fig. 2b) and radial cracks (Fig. 2c).

Wrinkling occurs due to high compressive circumferential stress $\sigma_{c}$ (Fig. 1) buckling the flange [6]. To avoid wrinkling, a combination of tensile and compressive stresses in the material needs to be introduced gradually. Therefore the conventional spinning must be carried out as multi-pass conventional spinning $[4,7]$. Furthermore, high stress in any direction is undesirable. Excessive tensile radial stress $\sigma_{r}$ (Fig. 1) may cause circumferential cracks. Radial cracks may form in two different cases [2]: due to circumferential tensile stresses or a combination of circumferential compressive stresses and bending stresses which occur when existing wrinkles are being worked.

Range of occurrence these undesirable phenomena in the spinning process depends on many factors, e.g., mechanical properties and plastic properties of material, structure, technological parameters of the process, number of passes of spinning rollers, geometry of the roll and feed, the template, trajectory of the rollers in each crossing, etc. Knowledge of the incidence of these adverse phenomena limiting the possibility of forming sheet metal in this process is especially important when design- ing industrial processes carried out on spinning machines of various design.

The aim of this work was to determine the relationship between the essential parameters of the spinning process described later in this work. Determined relationship is a useful tool in the design process of spinning pieces from alloy AMS 5504 under industrial conditions. Developed relationship enables an initial assessment of the possibility of forming the expected products in the actual conditions of the process of spinning due to the possibility of limiting phenomena: wrinkling and material cracking.

\section{Technological indices in the spinning process}

Technological indices characteristic for the given process are parameters that are of particular importance during the development of the technological process. On their basis the technologist is able to make a discernment on the scope and feasibility of an item or a semi-finished product by a specific manufacturing technology.

The main technological parameter which affects significantly the course of the spinning process is the dimensionless deformation coefficient or the spinning coefficient $b$, defined as:

$$
\beta=\frac{D}{d}
$$

where: $D$ - initial diameter of the sheet, $d$ - diameter of the drawpiece.

Generally speaking, with increasing values of this coefficient, the spinning process becomes more technologically complex, primarily because of the difficulty in determining the optimum number of passes of the forming roller and its trajectory in the individual passes. However, when at the limit value of this coefficient $\beta=\beta_{g r}$, the spinning process cannot be realized in the given conditions, due to the occurrence of phenomena which restrict the scope of the techniques of spinning, i.e. wrinkling or cracking of the shaped material.

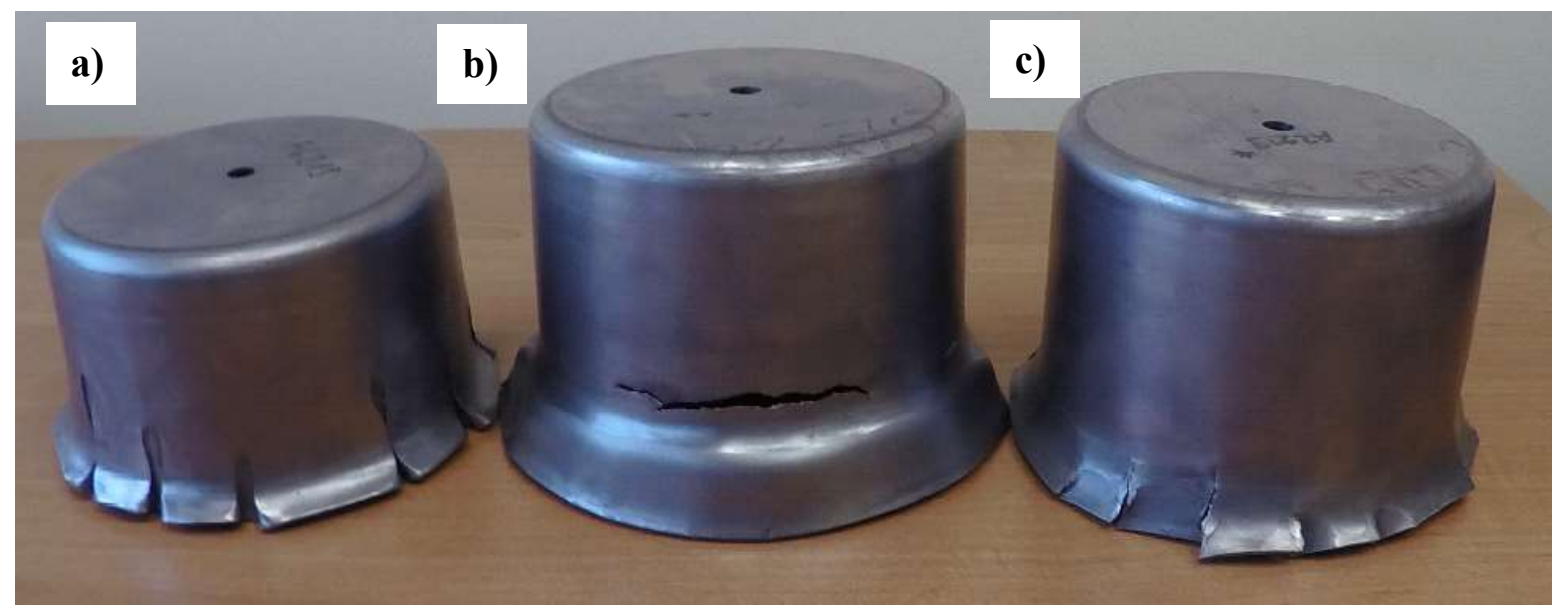

Fig. 2. The most common defects in spinning as a result of adverse events limiting the spinning process: a) wrinkling, b) circumferential cracks, c) radial cracks 
However, in engineering practice the use of the spinning ratio is not sufficient as it does not take into account of the thickness of the formed sheet. The thickness of the sheet has a very significant effect on the occurrence of phenomena which limit the spinning process. Admittedly with an increase of the sheet thickness its tendency for wrinkling decreases, but its propensity for cracking and thinning out grows.

An influence of the sheet thickness during the spinning is considered by so-called thin-wall ratio defined as:

$$
\gamma=\frac{t_{0}}{d}
$$

where: $t_{0}$ - initial sheet thickness, $d$ - template diameter.

The authors of the papers [8-10] showed that the limiting spinning ratio $\beta_{g r}$ depends strongly on the thin-wall ratio $\gamma$ :

$$
\beta_{g r}=f(\gamma)
$$

Knowledge of this relationship determined experimentally for the specific conditions of the spinning process is very useful in engineering practice. Its knowledge allows to evaluate the possibility of a correct execution of the drawpiece using the specified parameters of the spinning process.

In the further part of this article the experimental studies were presented which aimed at determination of the dependence of the limiting spinning coefficient $\beta_{g r}$ on the sheet wall thickness using two different rounding radii of forming rollers.

\section{Experimental research}

Experimental research was carried out on a two-roller industrial spinner of a vertical axis Leifeld SFC 800 V500 which is owned by Pratt \& Whitney Rzeszow. The working station for spinning tests with a visible arrangement of forming tools was shown in Fig. 3. For the research the station was equipped with the following tools: a) a mandrel (1) with a diameter $d=122.5 \mathrm{~mm}$ and a maximum working height $h_{\max }=190 \mathrm{~mm}$; b) two sets of forming rolls (2) of outer diameter $D_{r}=320 \mathrm{~mm}$ and working radii $r=(6$ and 12$) \mathrm{mm}$. In relation to the mandrel

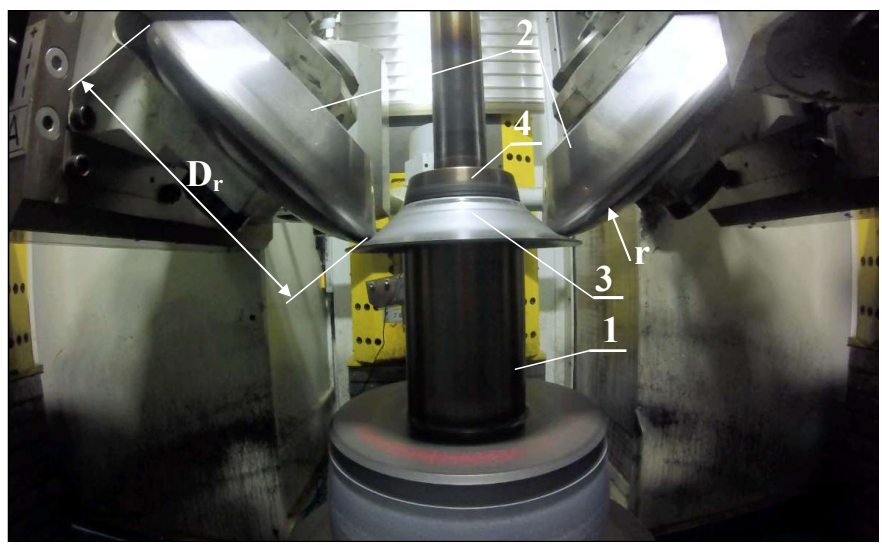

Fig. 3. The working station for spinning tests: 1 - mandrel, 2 - rolls shaping, 3 -sheet, 4 -tailstock (Pratt \& Whitney Rzeszów S. A. proprietary) axis the forming rollers are inclined at a constant angle of $45^{\circ}$. The CNC spinner enables the control of tools motion during spinning from the program or manually. It is also possible to generate a control program based on manual control, and then its start.

Relation determination $\beta_{g r}(3)$ requires the implementation of spinning tests using various values of spinning coefficient $\beta$ (1) and the thin-wall ratio $\gamma(2)$. Different values of spinning coefficient can be achieved using a mandrel with a constant diameter and sheet metal discs with different diameters, or by using sheet metal discs with constant diameters and mandrels with several different diameters. However, due to the necessity of the condition fulfillment $(r / d)=$ const in determining the relation (3) the mandrel with a constant diameter $d=122.5 \mathrm{~mm}$ and the sheet metal discs of suitably different diameters were used. The diameters of the respective discs for the research were calculated according to the formula:

$$
D_{i}=\beta_{i} \cdot d
$$

where: $D_{i}$ - successive diameter of discs for spinning, $\beta_{i}=$ $(1.1,1.2,1.3,1.4,1.5 \ldots .$.$) - spinning coefficient, which was in-$ creased for the next disc by a value $0.1, d$-diameter of template.

The diameter of the discs for testing was increased until the limit values of spinning coefficient was achieved, i.e. after which during the spinning process wrinkling or the cracking of the shaped material occurred.

For the tests the sheet discs of the metal thickness $t_{0}=(0.88,1.56,1.96$ and 3.1$) \mathrm{mm}$ were used. Such a selection of sheet thickness allowed to get the thin-wall ratio $\gamma$ in the range which is the most frequently used in industrial practice.

For the tests the sheet AMS 5504 was chosen. Stainless steel AISI 410, EN 1.4006 is a basic hardenable martensitic stainless steel and is well suited for use in highly stressed parts where properties such as corrosion resistance, good strength and ductility are required. A variety of sheet metal parts i.e.: aircraft engines and gas turbines are made of this type of material.

The spinning process was carried out using a constant rotational speed of the spinner spindle $n=500 \mathrm{rpm}$ and the feed rate of the forming rolls $p=300 \mathrm{~mm} / \mathrm{min}$. The tests were conducted using the forming rolls of rounding radii $r=6 \mathrm{~mm}$ and $r=12 \mathrm{~mm}$ (Fig. 3). The arc concave trajectory of tools was used, which with comparison to the arc-convex and linear trajectory allows to obtain the biggest spinning coefficient [11]. Working movement was only performed by the roller on the edge of the disc, so without forming on return. The number of passes of forming rollers for the spinning coefficient was the same and grew up with an increase of the spinning coefficient values. The applicable number of passes of forming rollers depending on the spinning coefficient is shown in Table 1. The simplified algorithm for the course of the conducted tests was presented schematically in Fig. 4.

The discs for research of diameter selected according to the equation (4) were cut by a laser from the sheet of the given thicknesses. All discs were marked appropriately, and their edges after laser cutting were cleaned mechanically. The spinning tests started from a spinning disk of the smallest diameter and 
TABLE 1 thickness. Then, in subsequent attempts a spinning ratio was

The number of passes of forming rollers depending on the spinning coefficient

\begin{tabular}{|c|c|c|c|}
\hline \hline $\begin{array}{c}\text { Spinning ratio } \\
\boldsymbol{\beta}\end{array}$ & $\begin{array}{c}\text { Number of } \\
\text { passes } \\
\boldsymbol{i}\end{array}$ & $\begin{array}{c}\text { Spinning ratio } \\
\boldsymbol{\beta}\end{array}$ & $\begin{array}{c}\text { Number of } \\
\text { passes } \\
\boldsymbol{i}\end{array}$ \\
\hline 1.1 & 1 & 1.6 & 6 \\
\hline 1.2 & 2 & 1.7 & 7 \\
\hline 1.3 & 3 & 1.8 & 8 \\
\hline 1.4 & 4 & 1.9 & 9 \\
\hline 1.5 & 5 & 2.0 & 10 \\
\hline
\end{tabular}
same thickness until the wrinkling and/or cracks appeared on the drawpiece. Then for the ratio where the cracks appeared the tests were repeated twice more. If in subsequent attempts there were still defects on the drawpieces, the spinning ratio corresponding to the highest recorded value at which the defects in the form of wrinkling and/or cracks on the drawpiece did not appear was assumed as a limiting value for this thickness. After determining the values of the limiting spinning ratio for the smallest sheet thickness the same test procedure was carried out for spinning

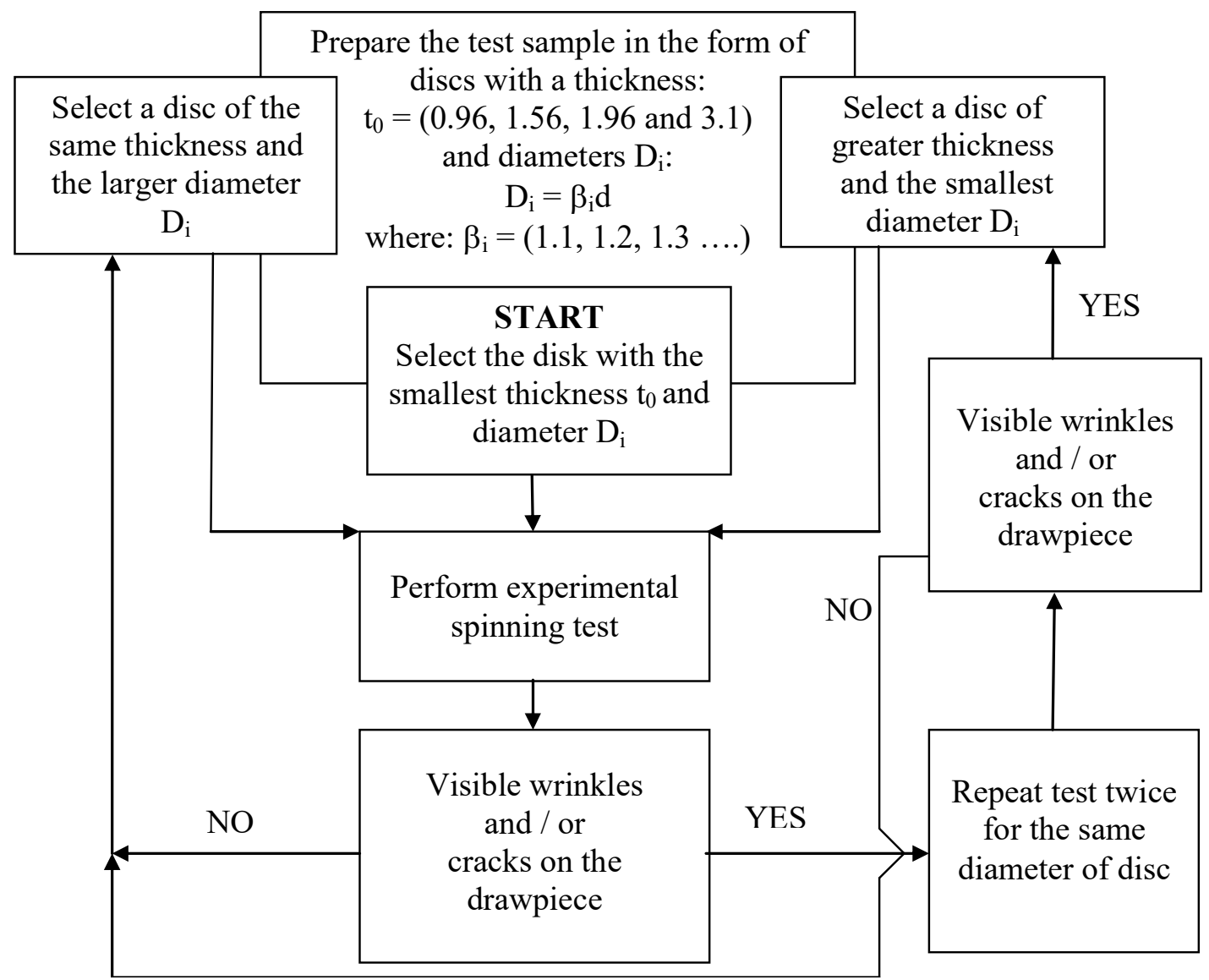

Fig. 4. The simplified algorithm of research

the discs from the sheet of greater thickness. The procedure was repeated for all the tested thicknesses. In this way, the tests were made with the use of spinning rollers of fillet radius $r=6 \mathrm{~mm}$, and the rollers of fillet radius $r=12 \mathrm{~mm}$.

\section{Research results and their analysis}

Based on the tests there were determined the values of the limiting spinning ratios depending on the applied thin-wall ratio for two different fillet radii of forming rollers. The obtained experimental dependence of the limiting spinning on the thin-wall factor for the tested fillet radii of the forming rollers are presented in graphical form on the charts (Fig. 5, Fig. 6).
The dependencies featured in charts show that, irrespective of the fillet radius of the forming rollers $r$, with the growth of the thin-wall ratio the value of the limiting spinning rate increases. In both cases, the most rapid growth of the limiting spinning ratio was observed for thin-wall ratio within the range of 0.0071 to 0.0127 . In the first range of the lowest thin-wall ratios, the phenomenon which limited the spinning process was the wrinkling of the drawpiece flange. An increase of the fillet radius of the forming rollers $r$, and thus the ratio $(r / d)$ in the considered case from 0.049 to 0.098 caused an increase in the limiting forming ratio. It can, therefore, be concluded that an increase of the quotient $(r / d)$ delays the occurrence the flange wrinkling, thus enables the formation of drawpieces without wrinkling within higher values of spinning ratios. 


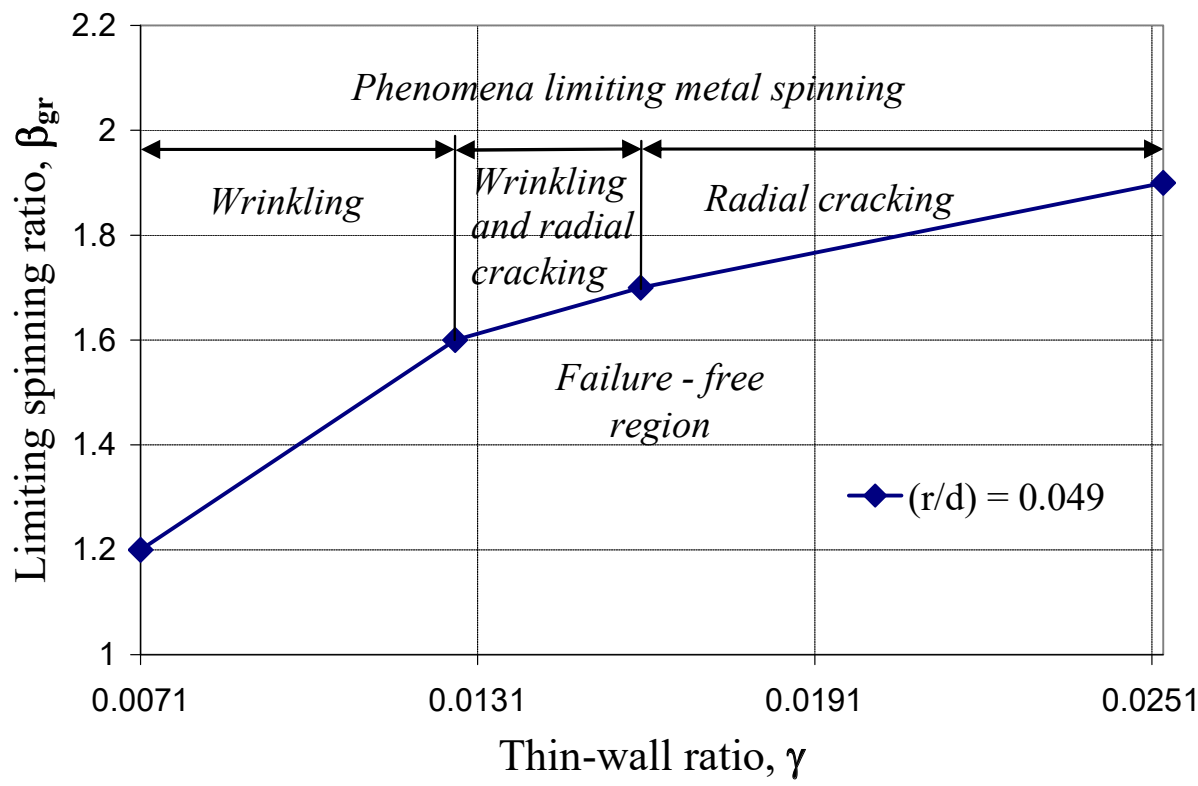

Fig. 5. Dependence between limiting spinning ratio and thin-wall ratio (AMS 5504, fillet radius of roller $r=6 \mathrm{~mm}$ )

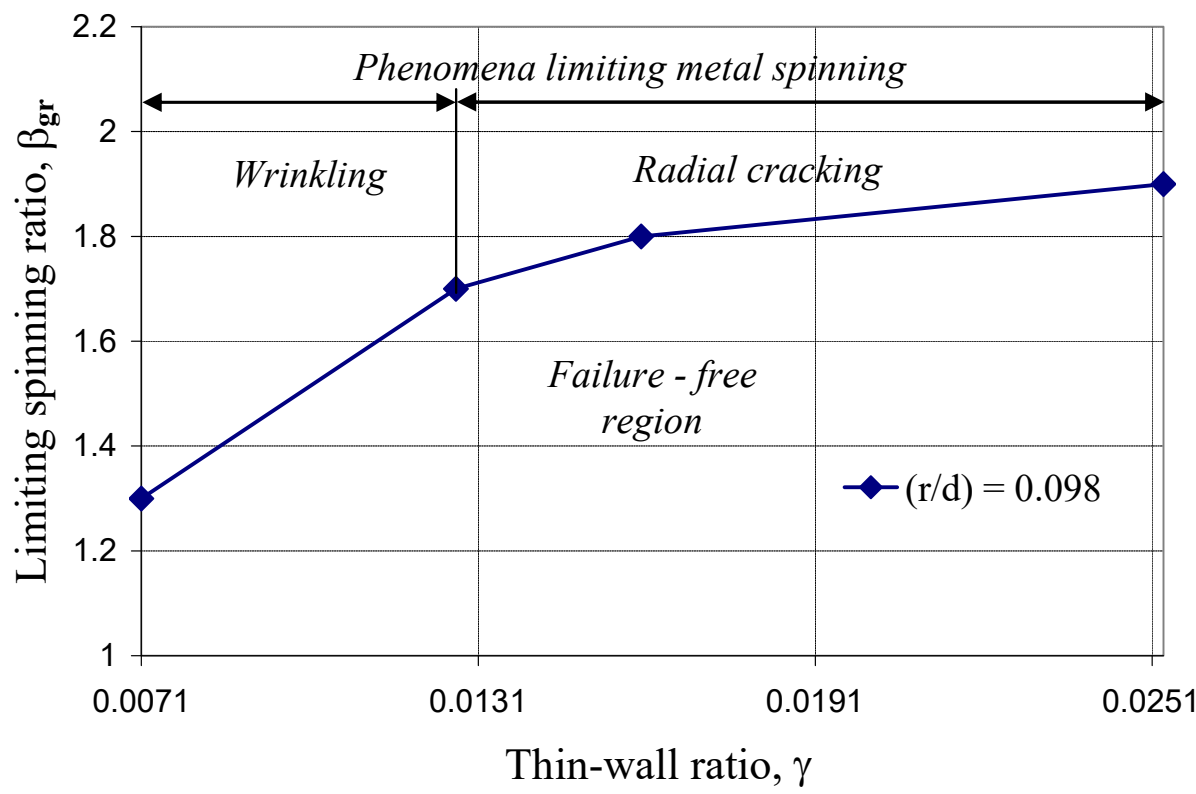

Fig. 6. Dependence between limiting spinning ratio and thin-wall ratio (AMS 5504, fillet radius of roller $r=12 \mathrm{~mm}$ )

In the second range of the tested thin-wall ratios from 0.0127 to 0.016 the phenomena limiting the spinning process depended on the quotient $(r / d)$. When using $(r / d)=0.049$ two limiting phenomena, i.e. wrinkling and radial cracking occurred. However, in the same range using $(r / d)=0.098$ the limiting phenomenon was only a circumferential cracking. It can, therefore, be concluded that an increase of $(r / d)$ causes not only an increase in the limiting spinning ratio, but also reduces the range of values of thin-wall ratio where the phenomenon of the drawpiece flange wrinkling occurs. As before, also in this range of thin-wall ratios the increase in $(r / d)$ improved the limiting spinning ratio.

In the third range of the examined thin-wall ratios from 0.016 to 0.0253 the phenomenon limiting both quotient $(r / d)=0.049$, as well as $(r / d)=0.098$ was radial cracking. In all the cases the cracks were initiated from the edge of the partially shaped disc. What is interesting for the thin-wall ratio $\gamma=0.0253$ the designated value of the limiting spinning ratio is the same for both the tested values of the quotient $(r / d)$. It should be noted that during forming the metal from the alloy AMS 5504 of 3.1 $\mathrm{mm}$ at a rate of spinning ratio of 1.9 , the forming forces were so large that the surface pressure by using rollers of a fillet radius $r=6 \mathrm{~mm}$ caused plasticization and the flow of the material on the contact surface with the roller. Accordingly, for this case there was obtained big thinning of the spinned sheet and more uneven (wavy) surface as compared to a spinning by a roller with a fillet radius $r=12 \mathrm{~mm}$. Thus, the phenomenon limiting the process apart from the wrinkling and cracking could be excessive thinning of the drawpiece wall which, in most parts of the sheet is allowed in a very narrow range of tolerances for the wall thickness of the drawpiece. 


\section{Conclusions}

Based on the survey, the following conclusions can be drawn:

1. The spinning ratio, the thin-wall ratio and the relation of the sheet thickness to the fillet radius of the forming roll are very important technological parameters characterizing the spinning process. Knowledge of the relationship between them and the significance of their impact on the process of spinning carried out under certain circumstances allows to take into account the range of adverse phenomena which, already at the design process, limit the spinning of the drawpiece of the required parameters.

2. With the growth of thin-wall ratio, the limiting spinning ratio grows as well. The increase in the limiting spinning ratio is more intense in the range of small thin-wall ratios, i.e., those for which the flange wrinkling is the phenomenon limiting the spinning process.

3. Virtually in the whole range of the applied thin-wall ratios, the limiting spinning ratio is depends on the quotient $(\mathrm{r} / \mathrm{d})$. Larger values of the limiting spinning ratio were obtained for $(r / d)=0.098$ than $(r / d)=0.049$. So we can generally say that with an increase in the value of the quotient $(r / d)$ the limiting spinning ratio grows as well.

4. The type of the phenomena which limits the spinning process depends on the value of the thin-wall ratio and the quotient $(r / d)$. In the present case, at lower values of this ratio, i.e. up to 0.0127 the phenomenon which limits the spinning process was to the wrinkling of the drawpiece flange, but for larger values of this ratio the limiting phenomenon was the radial cracking.

5. With the growth of thin-wall ratio and the spinning ratio increases the thinning of the drawpiece wall increases, whereby its size also depends on the quotient $(r / d)$. In the present case, lower thinning was observed at a higher value of this quotient, especially for the highest value of the thinwall ratio.

\section{Acknowledgements}

Financial support of the National Center for Research and Development within the INNOLOT program project No. INNOLOT/I/9/NCBR/2013 CASELOT is gratefully acknowledged.

\section{REFERENCES}

[1] C.C. Wong, T.A. Dean, J. Lin, A review of spinning, shear forming and flow forming processes, Int. J. Mach. Tool. Manu. 43, 14191435 (2003).

[2] O. Music, J.M. Allwood, K. Kawai, A review of the mechanics of metal spinning, J. Mater. Process. Tech. 210, 3-23 (2010).

[3] J. Brown, Advanced Machining Technology Handbook, 1998 McGraw-Hill.

[4] M. Runge, Spinning and flow forming [Pollitt DH, Trans.], 1994 Leifeld $\mathrm{GmbH}$.

[5] L. Wang, H. Long, Investigation of material deformation in multi-pass conventional metal spinning, Mater. Design. 32, 2891-2899 (2011).

[6] M. Watson, H. Long, Wrinkling failure mechanics in metal spinning, Procedia Engineering 81, 2391-2396 (2014).

[7] K. Lange, Handbook of Metal Forming, 1986 SME Publications.

[8] E. Sieble, K.H. Dröge, Krafte und MateriatfluB beim Druken,Werkstants- technick und Maschinenbau 45 (1), 6-9 (1955).

[9] M. Hayama, T. Murota, On the study of metal spinning, Bull. Fac. Eng. 12, 53-88 (1963).

[10] K.I. Ahmed, M.S.Gadala, M.G. El-Sebaie, Deep spinning of sheet metals, Int. J. Mach. Tool. Manu. 97, 72-75 (2015).

[11] M. Hayama, H. Kudo, T. Shinokura, Study of the pass schedule in conventional simple spinning, Bulletin of the JSME 13 (65), 1358-1365 (1970). 